\title{
Personal motivation, self-regulation barriers and strategies for weight loss in people with overweight and obesity: a thematic framework analysis
}

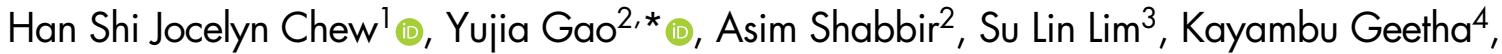 \\ Guowei Kim², Choon Seng Chong ${ }^{2}$, Davide Lomanto ${ }^{2}$ and Bok Yan Jimmy So ${ }^{2}$ \\ ${ }^{1}$ Alice Lee Centre for Nursing Studies, Yong Loo Lin School of Medicine, National University of Singapore, Singapore: \\ 2Department of Surgery, National University Hospital, National University Health System, Singapore, Singapore \\ 119228, Singapore: ${ }^{3}$ Dietetics Department, National University Hospital, National University Health System, \\ Singapore: ${ }^{4}$ Rehabilitation Department, National University Hospital, National University Health System, Singapore
}

Submitted 30 June 2021: Final revision received 14 December 2021: Accepted 14 February 2022: First published online 22 February 2022

\begin{abstract}
Objective: To explore motivations, self-regulation barriers and strategies in a multi-ethnic Southeast Asian population with overweight and obesity.

Design: Qualitative design using semi-structured face-to-face and videoconferencing interviews. Data were analysed using thematic framework analysis and constant comparison method.

Setting: Specialist weight management clinic.

Participants: Twenty-two participants were purposively sampled from 13 April to 30 April 2021. Median age and BMI of the participants were 37.5 (interquartile range $(\mathrm{IQR})=13.3)$ and $39.2 \mathrm{~kg} / \mathrm{m}^{2}(\mathrm{IQR}=6 \cdot 1)$, respectively. And $31.8 \%$ were men, majority had a high intention to adopt healthy eating behaviours (median $=6.5$; $\mathrm{IQR}=4 \cdot 8-6 \cdot 3$ ) and $59 \%$ of the participants had a medium level of self-regulation. Results: Six themes and fifteen subthemes were derived. Participants were motivated to lose weight by the sense of responsibility as the family's pillar of support and to feel 'normal' again. We coupled self-regulation barriers with corresponding strategies to come up with four broad themes: habitual overconsumption - mindful self-discipline; proximity and convenience of food available - mental tenacity; momentary lack of motivation and sense of control - motivational boosters; and overeating triggers removing triggers. We highlighted six unique overeating triggers namely: trigger activities (e.g. using social media); eating with family, friends and colleagues; provision of food by someone; emotions (e.g. feeling bored at home, sad and stressed); physiological condition (e.g. premenstrual syndrome); and the time of the day.

Conclusions: Future weight management interventions should consider encompassing participant-led weight loss planning, motivation boosters and selfregulation skills to cope with momentary overeating triggers.
\end{abstract}

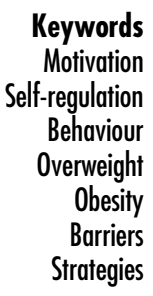

Overweight and obesity remains a persistent public health concern. In 2016, approximately $39 \%$ of the global adult population was reported to live with overweight and obesity, and this prevalence was expected to increase to $57 \cdot 8 \%$ by $2030^{(1,2)}$. While the prevalence rate in Southeast Asia (24.3\%) is relatively lower as compared to the USA (64.2\%) and Europe $(59.6 \%)^{(1)}$. Asian statistics could have also been underestimated by using the WHO's recommended BMI cut-off score of $>25 \mathrm{~kg} / \mathrm{m}^{2}$ as studies have shown that the Asian cut-off score should be lower at $>23 \mathrm{~kg} / \mathrm{m}^{2(3)}$. While there are guidelines for weight management ${ }^{(4)}$, studies have shown inertia towards providing clinical weight management services due to inadequate training in behavioural counselling and various treatment options ${ }^{(5)}$. Commonly proposed weight management strategies include calorie restriction, physical activity, behavioural modifications, pharmacological treatments and surgical interventions ${ }^{(6)}$. While the latter two strategies have shown higher weight loss effectiveness especially in the short term, lifestyle behaviour modification is often the 
first-line treatment, especially for people who are slightly overweight due to the lower risk of adverse effects ${ }^{(5)}$. However, behaviour change is well known to be challenging due to the complex system of obesity involving more than a hundred influencing factors such as food availability, environment, social-economic status, culture, psychology, just to name a few ${ }^{(7)}$.

Popular behavioural interventions for weight management include cognitive behavioural therapy ${ }^{(8)}$, motivational interviewing ${ }^{(9)}$, functional imagery training ${ }^{(10)}$, and diet and exercise counselling ${ }^{(11)}$. Other interventions are designed to improve motivation, mindfulness, cognitive restraint, emotional regulation, environmental modifications, and most importantly, self-regulation ${ }^{(12,13)}$. However, findings on their effectiveness on behaviour change and weight loss have been either inconsistent or unsustainable as participants often experienced weight regain $^{(6,14)}$. Moreover, existing interventions could be more targeted at individuals who are already motivated to engage in healthy weight loss behaviours, highlighting a lack of focus on motivation and self-regulation skills. Consistent with previous studies and the Temporal Selfregulation Theory, these two factors are crucial in resolving the 'psychological tension' developed between prepotent behaviours (e.g. habits and reflexes) and new intentional behaviour $^{(15,16)}$. One study also showed that adults with poor as compared to adequate eating self-regulation (i.e. ability to control eating behaviours to adhere to eating intentions) were 3.81 times more likely to be overweight or obese ${ }^{(17)}$. However, information on personal motivations, self-regulation barriers and facilitators for weight loss remain scarce. These are important information to develop person-centred interventions to enhance intervention effectiveness. To the authors' best knowledge, no qualitative studies on this topic in a multi-ethnic Southeast Asian society could be found. Based on a recent qualitative study, negative consequences of obesity were found to be a strong motivation for weight loss and self-regulation was found to enhance the sustainability of a healthy weight management routine ${ }^{(18)}$. However, this study was the only representative of eleven women who participated in a weight loss programme. In another study, physical activity was found to be motivated by hedonistic factors such as having positive socioenvironmental support and experiences. However, this study was only representative of twelve young women aged 18 to 21 years and was focused solely on physical activity that does not cover others aspects of weight loss. Therefore, the current study aimed to explore the weight loss motivations, self-regulation strategies and challenges in people with overweight and obesity. The specific questions were (1) What are the motivators of weight loss in people with overweight and obesity? and (2) What are the self-regulation barriers and strategies used by people with overweight and obesity?

\section{Methods}

\section{Study design and participant recruitment}

This qualitative study was conducted as part of a larger study. Participants were purposively sampled from a specialist weight management clinic in Singapore from 13 April to 30 April 2021. Face-to-face and videoconferencing (Zoom) interviews were conducted based on a semi-structured interview guide that was developed based on our literature review. Face-to-face interviews were conducted exclusively either at a consultation room or quiet area of the clinic's waiting area. Participants were recruited if they (1) were $>21$ years old, (2) have a BMI $\geq 23 \mathrm{~kg} / \mathrm{m}^{2}$, (3) were able to comprehend the English language and (4) were able to provide informed consent. Participants with severe psychiatric disorders were excluded. Twenty-seven potential participants were invited to participate in this study, three declined as they were busy and two were lost to videoconferencing interview. Findings are reported according to the Consolidated Criteria for Reporting Qualitative Research (COREQ) checklist (Appendix 1) ${ }^{(19)}$.

\section{Procedure}

Once the study information such as purpose and procedures were explained to the participants and informed consent was obtained, participants completed questionnaires on their sociodemographic profile, eating intentions, self-regulation (Self-regulation of Eating Behavior Questionnaire (SREBQ)) (20) and physical activity (International Physical Activity Questionnaire-Short Form $($ IPAQ-SF $))^{(21)}$. A total of twenty-two participants were purposively sampled for their ability to provide rich information about their motivations and self-regulation for weight loss. Data saturation was reached at the eighteenth participant where no new information emerged. Interviews were conducted by one interviewer (HSJC, PhD, a research fellow with more than 6 years of qualitative research experience) to ensure consistency. Strategies such as keeping a reflexivity journal and maintaining an audit trail were used to mitigate the potential risk of preconceived ideas influencing the qualitative research inquiry ${ }^{(22)}$. Interviews were audiotape-recorded, and the observational field notes on the participants' non-verbal cues were noted.

\section{Data management and analysis}

Interviews were stored on an internal cloud server, manually transcribed verbatim, and analysed thematically using the Nvivo software $12^{(23)}$. Analyses were conducted using the five-step thematic framework analysis and constant comparison method (i.e. simultaneous data collection and analysis within and between transcripts) ${ }^{(24,25)}$. The framework analysis method was chosen for the ease of within- and 
between-case comparisons, systematic treatment of analytical units, and the comprehensive nature of analysing the entire set of materials obtained ${ }^{(24,25)}$. HSJC first familiarised herself with the data by iteratively listening to the interview audiotape recordings and reading the interview transcripts. During this process of immersion, key ideas and concepts were also annotated and coded. Next, a thematic framework was inductively identified through the categorisation of emerging ideas into preliminary themes. In the third step, sections of data and codes were indexed under the respective themes. These themes were then charted into framework matrices to summarise the findings in a systematic and structured manner. Lastly, congruence and discrepancies across the themes were identified, interpreted and mapped. Resultant themes were discussed with all the co-authors and discrepancies were resolved through consensus. The interviewer practised bracketing during the data collection and analysis process to avoid introducing biases and assumptions that may cloud objective judgements.

\section{Results}

The median age of the participants was 37.5 years old (interquartile range $(\mathrm{IQR})=13.3$; range $25-68)$ and had a median BMI of $39 \cdot 2 \mathrm{~kg} / \mathrm{m}^{2}$ (IQR $=6 \cdot 1 ; 27 \cdot 8-50 \cdot 6$ ) (sample characteristics are detailed in another study). Majority of the participants had a high intention to adopt healthy eating behaviours (scale of $0-7$ ) (median $=6 \cdot 5$; IQR 4.8-6.3) and $59 \%$ of the participants had a medium level of eating self-regulation (Table 1). The number of dietitian and physiotherapist visits ranged from 1 to 17 and 1 to 6 , respectively, and the duration of follow-up since the index visit ranged from 1 week to 29 months (Table 2). No trend was identified between the number of dietitian and physiotherapist visits and self-regulation of eating behaviours and physical activity. The interviews lasted between 30 to 50 min with a total of 875 narratives coded. For the research question on the motives for weight loss, two themes and six subthemes emerged (Table 3). For the research

Table 1 Participant characteristics ( $n$ 22)

\begin{tabular}{lcc}
\hline Participant characteristics & Statistics & $\begin{array}{c}\text { Median, IQR, } \\
\text { range }\end{array}$ \\
\hline $\begin{array}{l}\text { Intention to adopt healthy eating } \\
\text { behaviours }\end{array}$ & 6.5 & $4 \cdot 8-6 \cdot 3$ \\
Level of eating self-regulation & & \\
$\quad$ Low & 6 & $27 \cdot 3$ \\
Medium & 13 & $59 \cdot 1$ \\
High & 3 & $13 \cdot 6$ \\
MET-min/week & 841.5 & 2107.9 \\
Level of physical activity & & \\
Low & 8 & $36 \cdot 4$ \\
Moderate & 9 & 40.9 \\
High & 5 & $22 \cdot 7$ \\
\hline
\end{tabular}

IQR, interquartile range; MET, metabolic equivalent task. question on the self-regulation barriers and strategies used for weight loss, four themes and ten subthemes emerged (Table 3). The twenty-two participants were anonymised by numerical pseudonyms, gender, age and race (e.g. P10, Man, 53, Chinese) (Table 2).

\section{Question 1: what are the participants' motives for weight loss?}

\section{Family's pillar of support}

Most of our participants were middle-aged adults who were often motivated by the need to care for their parents and children to stay healthy. One participant said

'To make sure that when drastic things happen, for example, touch wood, my father or my mom, fall in health, I will be there. I want to see my daughter grow up. I want to see my younger siblings achieve their dreams'. -P16, Woman, 35, Malay-

Participants also expressed that even if they were unable to provide for their family, the least that they could do was to avoid burdening them and to set a good example of living healthily. One participant said 'If heart attack and die straight away, it's ok. I'm more worried about stroke. I don't want to be a burden to my family physically, emotionally and financially'. -P4, Man, 42, Chinese- Another participant said, 'I want to also set a good example for them (children), good eating habits, good health'. -P14, Woman, 47, Indian-

\section{To feel 'normal' again}

Participants also mentioned being motivated by their desire to feel physically and aesthetically fit again to perform daily activities smoothly. To do so, they emphasised on three subthemes namely the need to: (1) feel less pain and breathlessness; (2) feel more energetic; and (3) feel more confident.

One participant said, 'The joints, starting to have aches and pains more often. Even wear socks I must sit at the edge of the sofa or bed. You ask me to bend down like that wear I cannot. Breathless'. -P4, Man, 43, Chinese- Others mentioned being motivated by the thought of having more energy to play with their children and perform at work. A participant said, 'I would want to be healthy to actually play along with him (son) ... Because both of us have a long way to go still'. -P7, Woman, 34, Malay-

Next, participant verbalised the sense of being discriminated at work and by the lack of clothes available in their sizes. One participant said, 'Although you might not be the lazy type, you might be the very active type, but then it's just the impression that you give people'. -P13, Woman, 39, Chinese- Another said, 'I actually cried. You know when you gain weight, it's also hard to get some clothes from the shop' -P8, Woman, 29, Malay- These highlighted the daily challenges faced by the participants, nuanced as negative emotions such as disappointment, frustration and helplessness. 


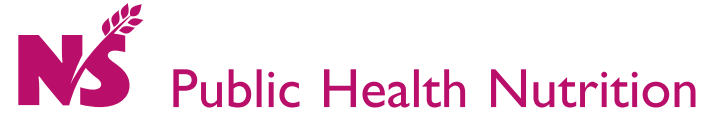

Table 2 Participants' individual characteristics

\begin{tabular}{|c|c|c|c|c|c|c|c|c|c|c|c|}
\hline $\begin{array}{l}\text { Subject } \\
\text { code }\end{array}$ & Age & Gender & Race & $\begin{array}{l}\text { Highest } \\
\text { education level }\end{array}$ & $\begin{array}{l}\text { Occupational } \\
\text { sector }\end{array}$ & $\begin{array}{l}\text { Date of bariatric } \\
\text { surgery (if } \\
\text { applicable) }\end{array}$ & $\begin{array}{l}\text { Duration of follow-up } \\
\text { since index visit (months) }\end{array}$ & $\begin{array}{l}\text { Number of } \\
\text { dietitian visits }\end{array}$ & $\begin{array}{c}\text { Number of } \\
\text { physiotherapist } \\
\text { visits }\end{array}$ & $\begin{array}{l}\text { Level of eating } \\
\text { self-regulatory } \\
\text { skills }\end{array}$ & $\begin{array}{l}\text { Physical } \\
\text { activity } \\
\text { category }\end{array}$ \\
\hline P2 & 48 & Woman & Indian & $\begin{array}{l}\text { Secondary } \\
\text { school }\end{array}$ & NA & 2015 & 17 & 17 & 2 & Medium & Low \\
\hline P3 & 42 & Man & Arab & University & Finance & 2019 & 29 & 8 & 6 & Medium & Moderate \\
\hline P4 & 43 & Man & Chinese & University & Healthcare & - & .25 & 1 & 1 & Medium & Moderate \\
\hline P5 & 27 & Woman & Chinese & $\begin{array}{l}\text { ITE/Polytechnic/ } \\
\text { Junior college }\end{array}$ & Administration & 2017 & 53 & 6 & 0 & Medium & High \\
\hline P6 & 68 & Man & Malay & $\begin{array}{l}\text { Secondary } \\
\text { school }\end{array}$ & Retail/security/ & manufacturing & - & 33 & 3 & 1 & Low \\
\hline \multicolumn{12}{|l|}{ Low } \\
\hline P7 & 34 & Woman & Malay & $\begin{array}{l}\text { ITE/Polytechnic/ } \\
\text { Junior college }\end{array}$ & Childcare & - & - & 1 & 1 & High & Low \\
\hline P8 & 29 & Woman & Malay & $\begin{array}{l}\text { Secondary } \\
\text { school }\end{array}$ & Administration & - & - & 1 & 1 & Medium & Moderate \\
\hline P10 & 32 & Woman & Malay & $\begin{array}{l}\text { ITE/Polytechnic/ } \\
\text { Junior college }\end{array}$ & Retail/security/ & manufacturing & 2021 & 7 & 8 & 2 & Medium \\
\hline \multicolumn{12}{|c|}{ Jurnor conlege } \\
\hline P11 & 36 & Man & Eurasian & $\begin{array}{l}\text { ITE/Polytechnic/ } \\
\text { Junior college }\end{array}$ & Finance & 2021 & 4 & 4 & 2 & Low & Low \\
\hline P12 & 29 & Woman & Malay & $\begin{array}{l}\text { Secondary } \\
\text { school }\end{array}$ & Childcare & 2021 & 4 & 6 & 3 & Medium & Moderate \\
\hline P13 & 39 & Woman & Chinese & University & Administration & 2020 & 6 & 2 & 1 & Medium & High \\
\hline P14 & 47 & Woman & Indian & $\begin{array}{l}\text { ITE/Polytechnic/ } \\
\text { Junior college }\end{array}$ & Healthcare & - & - & 1 & 1 & Medium & Moderate \\
\hline P15 & 44 & Man & Chinese & $\begin{array}{l}\text { ITE/Polytechnic/ } \\
\text { Junior college }\end{array}$ & $\begin{array}{l}\text { Engineer/ } \\
\text { technician }\end{array}$ & - & - & 1 & 0 & Low & Low \\
\hline P16 & 25 & Woman & Malay & $\begin{array}{l}\text { Secondary } \\
\text { school }\end{array}$ & Childcare & - & 2 & 1 & 1 & Low & Low \\
\hline P17 & 35 & Woman & Chinese & University & Administration & 2021 & 7 & 6 & 2 & Medium & Moderate \\
\hline P18 & 57 & Woman & Indian & $\begin{array}{l}\text { Secondary } \\
\text { school }\end{array}$ & NA & 2020 & 24 & 9 & 1 & High & Moderate \\
\hline P19 & 39 & Woman & Malay & $\begin{array}{l}\text { Secondary } \\
\text { school }\end{array}$ & NA & - & 2 & 1 & 1 & Medium & Moderate \\
\hline P20 & 30 & Man & Chinese & $\begin{array}{l}\text { ITE/Polytechnic/ } \\
\text { Junior college }\end{array}$ & $\begin{array}{l}\text { Engineer/ } \\
\text { technician }\end{array}$ & - & - & 1 & 1 & Medium & High \\
\hline P21 & 26 & Woman & Indian & University & Healthcare & - & - & 0 & 1 & Low & High \\
\hline P22 & 36 & Woman & Malay & $\begin{array}{l}\text { ITE/Polytechnic/ } \\
\text { Junior college }\end{array}$ & Healthcare & - & - & 1 & 1 & Medium & Low \\
\hline P23 & 39 & Man & Chinese & University & Finance & 2021 & - & 6 & 2 & High & Low \\
\hline P24 & 44 & Woman & Indian & University & Healthcare & - & - & 1 & 1 & Low & Moderate \\
\hline
\end{tabular}


Table 3 Themes and subthemes

\begin{tabular}{|c|c|}
\hline Themes & Subthemes \\
\hline \multicolumn{2}{|l|}{ Question 1: what are the motives for weight loss? } \\
\hline Family's pillar of support & $\begin{array}{l}\text { To look after children and parents } \\
\text { To avoid burdening the family } \\
\text { To set a good example for the family }\end{array}$ \\
\hline To feel 'normal' again & $\begin{array}{l}\text { To feel less pain and breathlessness } \\
\text { To feel more energetic } \\
\text { To feel more confident }\end{array}$ \\
\hline \multicolumn{2}{|l|}{$\begin{array}{l}\text { Question 2: what are the self-regulation barriers and strategies used } \\
\text { for weight loss? }\end{array}$} \\
\hline Habitual overconsumption - mindful self-discipline & $\begin{array}{l}\text { Being mindful of satiety and satisfaction } \\
\text { Substituting food items with low-calorie options } \\
\text { 'Tricking the mind' } \\
\text { Creating a routine/habit }\end{array}$ \\
\hline Proximity and convenience of food available - mental tenacity & $\begin{array}{l}\text { Perceived monetary reward from abstinence } \\
\text { Willpower to stay disciplined }\end{array}$ \\
\hline Momentary lack of motivation and sense of control - motivational boosters & $\begin{array}{l}\text { Having a role model } \\
\text { Witnessing others suffer from health complications }\end{array}$ \\
\hline Eating triggers - removing triggers & $\begin{array}{l}\text { Staying occupied } \\
\text { Out of sight, out of mind }\end{array}$ \\
\hline
\end{tabular}

\section{Question 2: what are the self-regulation barriers and strategies used for weight loss?}

Each theme was intentionally crafted with the emergent self-regulation barrier as themes and corresponding selfregulation strategies as subthemes.

\section{Habitual overconsumption - mindful self-discipline}

All participants knew the importance of calorie restriction and physical activity for weight loss but often described the inability to control eating habits. One participant said 'I cannot change the habit. The more things you forbid, it becomes a craving at the end. There's no stopping. If I have that temptation, I will go look for it. I will travel for it'. -P3, Man, 42, Arab- Such habits were commonly manifested as overconsumption due to calorie-dense food options, excessive meal portions, excessive eating frequencies and inappropriate meal timings.

Strategies used to regulate eating habits were (1) being mindful of satiety and satisfaction, (2) substituting food items with low-calorie options, (3) 'tricking the mind' and (4) creating a routine/habit.

One participant said 'When I go order stuff outside I don't jia fan (add rice), jia mian (add noodles). I eat already, satisfy the craving then move on'. -P20, Man, 30, ChineseLow-calorie food options like vegetables and water were commonly used to substitute meal items, especially carbohydrates, as an essential part of Asian cuisines. An interesting strategy identified was to 'trick the mind' as one participant said 'It's our mind, trick our mind. I will think as long as our mind (thinks we're) full, it's ok'. -P7, Woman, 34, Indian- Another participant said, 'I would imagine that my tomato is my ice cream, all my sweet stuff'. -P23, Man, 39, Chinese- However, low-calorie substitutions were sometimes unsustainable as one participant said, 'I believe I need that carbohydrate to sustain. I tried solely vegetable only but very fast go hungry. After that, I tried salad and protein. But it doesn't sustain me long enough'.
-P4, Man, 43, Chinese- Participants also mentioned regulating their diet through the creation of diet and exercise routines/habits such as meal prepping and designating meal and exercise timing. One participant said 'The whole week I only eat wrap. Either subway wrap or I do my wrap... Last meal is before 8 o'clock'. -P8, Woman, 29, Malay- However, for participants who were working shifts, creating a routine was deemed challenging as one participant mentioned, 'Like I tried meal prepping also but it's so like uncertain'. P21, Woman, 26, Indian- One participant tackled this problem by 'stealing time' to integrate physical activity into their work. 'The only time that I have is to steal time. So like diaper change, I do (participant works in the childcare sector). Whatever that can make my body move I will do it'. -P12, 29, Woman, Malay-

\section{Proximity and convenience of food available - mental tenacity}

Besides the internal self-regulation barrier of habitual overconsumption, participants highlighted the external barrier of food being too accessible. This was commonly due to the proximity of food and grocery stores nearby and convenient access to food at home, especially for those working from home during the COVID-19 pandemic or having a desk-bound job. One participant said

'Previously when I was staying in a private estate, it was about 15 min walk to the nearest NTUC (grocery store) and then it just wasn't worth the effort? Now I've moved to HDB and just 2 min, it's a coffee shop that's $24 \mathrm{~h}$... Beside the coffee shop, there is a Sheng Siong (grocery store). So usually I'll go there at night to do some small grocery shopping. Then I would just you know buy one bao (bun) whenever I see the coffee shop'. -P11, Man, 36, Chinese-

Another participant said 'Because you are at home right, so you keep eating and eating'. -P8, Woman, 
29, Malay- Moreover, working from home was described to promote a sedentary lifestyle that makes weight loss more challenging. 'So, it's like I don't even get up to walk you know when you go to work you either take a bus or drive right'. -P15, 44, Man, Chinese- Participants working the night shifts also highlighted the difficulty in obtaining healthy food options at night. One participant said 'Only during the day you can have a lot of (healthy) choices. Night you don't have. Then when you really feel hungry, you buy all those tidbits'.-P6, Man, 86, Malay-

To control the temptation to buy food items out of convenience, participants mentioned the use: (1) perceive monetary reward from abstinence and (2) willpower to stay disciplined. One participant said 'I just tell myself I don't have enough money (to buy unhealthy food), you know you just keep it for something else'. -P7, female, 34, Malay- Another participant said, 'That one we have to force yourself. If you want to be healthy you must force'. -P2, Woman, 48, Indian-

\section{Momentary lack of motivation and sense of control - motivational boosters}

Though all participants had intrinsic motivations for weight loss, these were often temporally distal and abstract as compared to immediate concrete temptations. This led to a momentary lack of motivation and a sense of control, which was commonly exacerbated by memories of failed weight loss attempts such as the use of fad diets, exercise, slimming pills, slimming centres and traditional Chinese medicine. One participant said 'Acupuncture, slimming centre, protein shake, then I tried on like just no carbo diet, but nothing works. That time I felt quite depressed and there was not much motivation to carry on'. -P23, Man, 39, Chinese- Moreover, almost half of the participants also mentioned that being overweight since young contributed to the lack of confidence in being able to lose weight successfully. Another reason for the momentary lack of motivation was the discouraging and judgemental remarks that evoked negative emotions. One participant mentioned 'So, whenever I tell them (family) must motivate me not to eat so much, they will look at me, you sure, you can? It's always like that. My family can be very demoralizing'. P16, Woman, 35, Malay- Another said, 'If you go hawker centre, less rice, then they will judge you. Might as well just buy a normal portion'. -P5, Woman, 27, Chinese-

Motivation was highlighted to be a volatile and transient construct that requires constant reinforcements and reminders. Besides the intrinsic motivations identified earlier, participants mentioned the importance of having motivational boosters in the form of (1) having a role model and (2) witnessing others suffer from health consequences. One participant said 'My husband has started very strict last 2 months and I plan to follow then let's see. He's doing very regularly, so I find that very inspirational for me'. -P24, Woman, 44, Indian- Another participant mentioned
'I realized many of my clients my age are slowly coming up with health issues and of course, it gave me a shock. So it just made me think that before I hit a point of no return, I better do something about it'. -P11, Man, 36, Chinese-

\section{Overeating triggers - removing triggers}

Six broad categories of overeating triggers were identified (Table 2). (1) Activities - scrolling through social media (exposing one to visual cues of appealing food items through food posts: recipes, cooking shows, and eating shows and advertisements), watching shows on the television (habit of eating while enjoying a show and visual cues from food advertisements), eating (appetiser that leads to overeating), shopping (sense of novelty and the fear of missing out upon visual food stimuli), special occasion (sense of novelty and the fear of missing out upon visual food stimuli) and smoking cessation; (2) eating with family, friends and colleagues; (3) provision of food by someone; (4) emotions - feeling bored at home, sad and stressed; (5) physiological condition - feeling cool especially on rainy days, experiencing premenstrual syndrome and feeling tired after many activities; and (6) time of the day $-3-4 \mathrm{pm}$ at work and night. It is noteworthy that a trigger for one participant may be the inhibitor for another. For example, while some participants tend to eat more with friends as they can order more and try a wider variety of food items, others claim to eat less with friends in fear of being judged as a glutton (Supplement 1). Some also claim to eat more due to the sociocultural upbringing of not wasting food. One participant said 'I'm brought from young that I'm not supposed to waste food. So I eat everything. Because there's a lot of food and I don't want to waste. That's the main reason. So until I really full then I will stop'. P4, Man, 43, Chinese- Due to the comprehensive details on each trigger, respective quotes are shown in Supplement 3.

Despite the extensive number of triggers expressed by the participants, only two strategies were used to remove these triggers: (1) staying occupied and (2) out of sight, out of mind. Participants said 'Keep myself busy. Actually, mainly doing something else will help'.-P13, Woman, 39, Chinese- 'I try to avoid seeing advertisements. And it does help'. -P15, 44, Man, Chinese- 'Nowadays I don't find any tidbits or snacks to put at home'. -P23, Man, 39, Chinese. However, there seems to only address the triggers of being bored at home and the triggers related to visual cues.

\section{Discussion}

To our best knowledge, this is the first study that explored personal motivations, self-regulation barriers and strategies for weight loss in a multi-ethnic Southeast Asian adult population with overweight and obesity. Majority of the participants were found to have a high intention to eat 
healthily but a medium level of self-regulation over consumption behaviours. Most of the participants reported a low (36.4\%) and medium (40.9\%) level of physical activity. These quantitative findings suggest that despite a high desire to eat adopt healthy eating habits, participants lacked the self-regulation to control their diet and exercise habits.

Similar to previous research ${ }^{(18,26)}$, participants were motivated by the health concerns associated with overweight and obesity and the presence of family support. In addition to having family support, we found that the sense of responsibility as a pillar of support for the family served as a strong motivator for weight loss. This coincides with a previous study on Singaporean patients with heart failure where the consideration of a family's future wellbeing was found to be a strong motivator of heart failure self-care behaviours ${ }^{(27)}$. This could be associated with the cultural importance of being able to provide for one's family in a Southeast Asian collectivist culture as compared to a more individualistic culture in Western countries ${ }^{(28)}$. Next, the motivation of feeling 'normal' again also coincides with a previous study where participants with overweight and obesity were reportedly motivated to lose weight to feel more 'comfortable with their body' (e.g. in the clothes they wore) ${ }^{(29)}$. We extend this finding that beyond wanting to feel aesthetically fitting with the social norms and standards, participants wanted to feel physiologically 'normal' in terms of agility, not feeling breathless so easily, and feeling fewer aches and pain caused by excessive weight gain and age. Interestingly, fitting into the societal standards of body image was not a strong motivation in our population as mentioned in another study conducted in Malaysia, despite sharing a similar Southeast Asian culture ${ }^{(30)}$. This could be due to the difference in the population studied, where the latter recruited only Malay participants from a homogenous occupation as government employees, while we recruited participants from various ethnicities (i.e. Chinese, Malay, Indian, others) of different occupations.

On the same note, participants in our study mentioned the need for momentary motivation boosters to tackle selfregulation barriers, possibly by having a role model and vicarious experience through others suffering from health complications. This furthers existing literature that often treats motivation as an antecedent of behaviour change when it should be repeatedly and momentarily evoked throughout one's translational process of change intention to actual behaviour change. Therefore, future weight management interventions could consider the use of technology such as mobile phone apps to provide timely reminders and prompts of one's motivation to lose weight, especially in developed countries with high mobile phone penetration $^{(12)}$. An example is the Lark Weight Loss Health Coach $A I$, an artificial intelligence enabled weight loss coaching and counselling programme, which reportedly resulted in a mean $2.38 \%$ of weight loss over 15 weeks $^{(31)}$.
However, researchers and developers should consider the perceptions and acceptability of such interventions and tailor the programmes accordingly to optimise engagement and interventional effectiveness in weight management ${ }^{(32)}$. This is especially when our findings showed various culturally specific motivators that should be tailored to each individuals' motivations, demographics and characteristics. Moreover, future studies could explore the temporal stability of such motivations to adjust the micro-interventional components accordingly as motivations may be transient as shown in our findings.

Self-regulation strategies such as self-monitoring, goalsetting, use of prompts and relapse prevention are well known to be associated with better interventional effects on behaviour change ${ }^{(33)}$. However, incorporating all potential strategies at random does not automatically increase the effectiveness of an intervention due to individual differences, and the personalised barriers and strategies used to overcome such barriers remain unclear ${ }^{(34)}$. We found that participants were aware of the need to control the quantity (portion) and quality (nutrition) of food but did not know how to control their unhealthy eating habits and cravings. All participants verbalised the essentiality of calorie restriction to lose weight but often cited the inability to regulate eating habits that led to overconsumption. This highlights the need for people with overweight and obesity to enhance self-regulation skills and not merely gaining self-regulation knowledge in overcoming self-regulation barriers. Notably, participants attributed large amounts of weight gain to the COVID-19 measure of working from home, where they had easy access to food without being judged for overeating in the public. This is a well-known phenomenon worldwide, which will inflate the prevalence of obesity in a postCOVID-19 era of more people working from home ${ }^{(35)}$. Similar to prior research ${ }^{(26,36-38)}$, unhealthy social eating, mindless eating, negative thoughts and emotions, receding motivation, food availability, and the lack of family support were found to be self-regulation barriers. However, our findings extend current knowledge on momentary selfregulation barriers by identifying six unique overeating triggers that lead to momentary dietary lapses, a factor that is often insufficiently addressed in current weight management programmes that often focus on providing patient education but not self-regulation skills training. From our study, participants only mentioned the use of two strategies to remove overeating triggers - by ignoring or removing visual cues such as food advertisements or cooking videos and staying occupied to prevent eating out of boredom. As momentary lapses are crucial starters of relapse, participants should be empowered with self-regulation skills to inhibit such trigger responses. Strategies could include the use of mobile phone-based just-in-time prompts ${ }^{(39)}$ or real-time progress feedback ${ }^{(40)}$ to regulate momentary trigger responses. Studies have shown that people with overweight and obesity are more likely to have cognitive 
inhibitory impairments, leading to a lower ability to control behavioural impulses ${ }^{(41)}$. Therefore, future studies could consider incorporating just-in-time adaptive interventions to assist in resisting food temptations at the right time, right location $^{(42)}$.

Consistent with previous studies, creating diet and exercise routines $^{(18)}$, mindful eating ${ }^{(43)}$ and food substitutions (i.e. eating vegetables and proteins led to lower consumption of unhealthy food) ${ }^{(44)}$ were common strategies used to overcome unhealthy eating habits. Interestingly, participants overcame impulsive food buying by rationalising the need to save money and stay disciplined. Understandably, behaviour change requires immense and continuous cognitive effort to resist every potential lapse before a new habit overrides an old one ${ }^{(45)}$. Therefore, weight loss programmes need to shift the focus away from prescribing diet and exercise regimens and towards the personalisation and timely modifications of such regimens to suit the participants' self-regulation capacity and goals.

\section{Strengths and limitations}

There were several strengths and limitations to this study. Firstly, we purposively sampled for participants from various ethnicities, age groups and education levels to enhance the representativeness of the study findings. This was essential in a multi-ethnic society like Singapore as different ethnicity brings along different cuisines that influence weight management. For example, Chinese food such as soup noodles tends to be high in $\mathrm{Na}$, while Malay and Indian food such as nasi lemak and Chicken Tikka Masala tend to be high in fats and carbohydrates. It is also common to celebrate special occasions with food, which was identified as an overeating trigger. Secondly, the trustworthiness of the findings was also enhanced through prolonged engagement, member-checking with selected participants, providing thick descriptions of each participants' characteristics, practising bracketing during every interview, having an audit trail and reflexivity journal to prevent researcher bias. However, although it would have been more rigorous for at least two coders to perform the thematic analysis independently, only one research was involved in it. Nevertheless, codes and quotes were discussed among the authors, and discrepancies were resolved upon discussion. Moreover, the majority of the participants were middle-aged adults who are in the sandwich generation of having to juggle work, taking care of children and parents ${ }^{(46)}$. This could have limited the transferability of our findings to people of other age groups.

\section{Implications for future research and practice}

We intentionally thematised our findings to be easily applied into interventions for future research and practice. For example, personalised motives identified could be incorporated into weight management plans through periodical prompts and reminders to sustain a long-term weight loss goal. This would be beneficial even if one has undergone brief counselling interventions such as motivational interviewing as such brief techniques may be unsustainable on their own ${ }^{(47)}$. We recommend future interventions to adopt a person-centred approach in developing each individual's weight loss plan, where participants take an active role in designing their weight loss plan with healthcare professionals facilitating the process and providing resources when needed. Future interventions could use our findings on self-regulation barriers and strategies to assess individual self-regulation challenges and recommend person-centred coping strategies. We would like to highlight the importance of targeting eating lapse triggers instead of overly focusing on prescribing diet and exercise recommendations and evoking transient motivations for weight loss. As mentioned, such strategies have already been shown to be effective but only to a limited extent due to the intention-behaviour gap. Habits, momentary overeating triggers and self-regulation capacity play huge roles in translating intention to behaviour change. Our findings on the specific triggers would be useful in the development of interventional components to target each trigger that commonly causes lapse behaviours of overconsumption, especially when one's willpower and motivation are weakened. As such interventions need to be just-in-time, researchers could consider the use of technology and handheld devices like mobile phones or wearables to provide timely prompts or other strategies.

\section{Conclusion}

While evoking intrinsic motivation is essential to start one's weight loss journey, the translation of an intention to actual behaviour change has to be built upon a scaffold of motivation boosters and self-regulation skills to cope with momentary distractions. To the our best knowledge, there are no other studies that identified personal motivation, self-regulation barriers and strategies in a multi-ethnic Southeast Asian population with overweight or obesity. More research is needed on strengthening self-regulation skills to cope with overeating triggers and cravings.

\section{Acknowledgements}

Acknowledgments: We thank the help from the Centre for Obesity Management and Surgery (COMS) at the National University Hospital, Singapore, for facilitating the patient recruitment and Javis Fung for assisting in the clinical data retrieval. Financial support: H.S.J.C. is supported by the Yong Loo Lin School of Medicine Start-Up Grant. Conflict of interest: The authors have no conflict to declare. 
Authorship: H.S.J.C.: conceptualisation, data curation, formal analysis, investigation, methodology, project administration, resources, software, supervision, validation, visualisation, writing - original draft, writing - review and editing. Y.G., A.S., S.L.L., K.G., G.K., C.S.C., D.L. and B.Y.J.S.: formal analysis, validation, writing - review and editing. Ethics of buman subject participation: This study was conducted according to the guidelines laid down in the Declaration of Helsinki, and all procedures involving research study participants were approved by the National Healthcare Ground Domain Specific Review Board (Ref: 2020/01439) and the study was registered with ClinicalTrials.gov (ref: NCT04833803). Written informed consent was obtained from all patients.

\section{Supplementary material}

For supplementary material accompanying this paper visit https://doi.org/10.1017/S136898002200043X

\section{References}

1. Chooi YC, Ding C \& Magkos F (2019) The epidemiology of obesity. Metabolism 92, 6-10.

2. Kelly T, Yang W, Chen C-S et al. (2008) Global burden of obesity in 2005 and projections to 2030. Int J Obes 32, 1431-1437.

3. WHO Expert Consultation (2004) Appropriate body-mass index for Asian populations and its implications for policy and intervention strategies. Lancet 363, 157-163.

4. Acosta A, Streett S, Kroh MD et al. (2017) White paper AGA: POWER - practice guide on obesity and weight management, education, and resources. Clin Gastroenterol Hepatol 15, 631.e10-649.e10.

5. Kushner RF (2018) Weight loss strategies for treatment of obesity: lifestyle management and pharmacotherapy. Progr Cardiovasc Dis 61, 246-252.

6. Olson K (2017) Behavioral approaches to the treatment of obesity. R I Med J 100, 21.

7. Vandenbroek IP, Goossens J \& Clemens M (2007) ForesightTackling Obesities: Future Choices Building the System Obesity Map. Government Office for Science, UK Government's Foresight Programme. www.foresight.gov. uk (accessed June 2021).

8. Castelnuovo G, Pietrabissa G, Manzoni GM et al. (2017) Cognitive behavioral therapy to aid weight loss in obese patients: current perspectives. Psychol Res Behav Manag 10, $165-173$.

9. Barrett S, Begg S, O'Halloran P et al. (2018) Integrated motivational interviewing and cognitive behaviour therapy for lifestyle mediators of overweight and obesity in community-dwelling adults: a systematic review and meta-analyses. BMC Public Health 18, 1-10.

10. Solbrig L, Whalley B, Kavanagh DJ et al. (2019) Functional imagery training $v$. motivational interviewing for weight loss: a randomised controlled trial of brief individual interventions for overweight and obesity. Int J Obes 43, 883-894.

11. Teeriniemi AM, Salonurmi T, Jokelainen T et al. (2018) A randomized clinical trial of the effectiveness of a Webbased health behaviour change support system and group lifestyle counselling on body weight loss in overweight and obese subjects: 2-year outcomes. J Intern Med 284, 534-545.

12. Chew HSJ, Ang WHD \& Ying L (2021) The potential of artificial intelligence in enhancing adult weight loss: a scoping review. Public Health Nutr 24, 1-59.

13. Puente JMG \& Martínez-Marcos M (2018) Overweight and obesity: effectiveness of interventions in adults. Enferm Clin 28, 65-74.

14. Dombrowski SU, Knittle K, Avenell A et al. (2014) Long term maintenance of weight loss with non-surgical interventions in obese adults: systematic review and meta-analyses of randomised controlled trials. BMJ 348, g2646.

15. Greaves C, Poltawski L, Garside R et al. (2017) Understanding the challenge of weight loss maintenance: a systematic review and synthesis of qualitative research on weight loss maintenance. Health Psychol Rev 11, 145-163.

16. Papies EK (2017) Situating interventions to bridge the intention-behaviour gap: a framework for recruiting nonconscious processes for behaviour change. Soc Personal Psychol Compass 11, e12323.

17. Balani R, Herrington H, Bryant E et al. (2019) Nutrition knowledge, attitudes, and self-regulation as predictors of overweight and obesity. J Am Assoc Nurse Pract 31, 502-510.

18. Thom G, Lean ME, Brosnahan N et al. (2021) 'I have been all in, I have been all out and I have been everything inbetween': a 2-year longitudinal qualitative study of weight loss maintenance. J Hum Nutr Diet 34, 199-214.

19. Tong A, Sainsbury P \& Craig J (2007) Consolidated criteria for reporting qualitative research (COREQ): a 32-item checklist for interviews and focus groups. Int J Qual Health Care 19, 349-357.

20. Kliemann N, Beeken RJ, Wardle J et al. (2016) Development and validation of the self-regulation of eating behaviour questionnaire for adults. Int J Behav Nutr Phys Act 13, 87.

21. Lee PH, Macfarlane DJ, Lam TH et al. (2011) Validity of the international physical activity questionnaire short form (IPAQ-SF): a systematic review. Int J Behav Nutr Phys Act 8, 115 .

22. Tufford L \& Newman P (2012) Bracketing in qualitative research. Qual Soc Work 11, 80-96.

23. QIP Ltd (2020) NVivo (Released in March 2020) https:// www.qsrinternational.com/nvivo-qualitative-data-analysissoftware/home (accessed July 2021).

24. Srivastava A \& Thomson SB (2009) Framework analysis: a qualitative methodology for applied policy research. J Adm Gov 4, 72-79.

25. Gale NK, Heath G, Cameron E et al. (2013) Using the framework method for the analysis of qualitative data in multidisciplinary health research. BMC Med Res Method 13, 1-8.

26. Al-Mohaimeed AA \& Elmannan AAA (2017) Experiences of barriers and motivators to weight-loss among Saudi people with overweight or obesity in Qassim region - a qualitative study. Open Access Maced J Med Sci 5, 1028.

27. Chew HSJ, Sim KLD \& Cao X (2019) Motivation, challenges and self-regulation in heart failure self-care: a theory-driven qualitative study. Int J Behav Med 26, 474-485.

28. Singh P, Campbell T, LeBlanc P et al. (2020) Development of a culturally tailored motivational interviewing-based intervention to improve medication adherence in South Asian patients. Patient Prefer Adherence 14, 757.

29. Surrow S, Jessen-Winge C, Ilvig PM et al. (2020) The motivation and opportunities for weight loss related to the everyday life of people with obesity: a qualitative analysis within the DO: IT study. Scand J Occup Ther 28(6), 479-487.

30. Ismail TAT, Jalil RA, Ishak WRW et al. (2018) Understanding dieting and previous weight loss attempts among overweight and obese participants: insights into my body is fit and fabulous at work program. Korean J Fam Med 39, 15.

31. Stein N \& Brooks K (2017) A fully automated conversational artificial intelligence for weight loss: longitudinal 
observational study among overweight and obese adults. JMIR Diabetes 2, E8590.

32. Chew HSJ \& Achananuparp P (2022) Perceptions and needs of artificial intelligence in healthcare to increase adoption: a scoping review. J Med Internet Res 24(1), e32939.

33. Lara J, Hobbs N, Moynihan PJ et al. (2014) Effectiveness of dietary interventions among adults of retirement age: a systematic review and meta-analysis of randomized controlled trials. BMC Med 12, 1-12.

34. Dombrowski SU, Sniehotta FF, Avenell A et al. (2012) Identifying active ingredients in complex behavioural interventions for obese adults with obesity-related co-morbidities or additional risk factors for co-morbidities: a systematic review. Health Psychol Rev 6, 7-32.

35. Chew HSJ \& Lopez V (2021) Global impact of COVID-19 on weight and weight-related behaviors in the adult population: a scoping review. Int J Environ Res Public Health 18, 1876.

36. Krukowski RA, West DS, Harvey-Berino J et al. (2010) Neighborhood impact on healthy food availability and pricing in food stores. J Community Health 35, 315-320.

37. Venditti EM, Wylie-Rosett J, Delahanty LM et al. (2014) Short and long-term lifestyle coaching approaches used to address diverse participant barriers to weight loss and physical activity adherence. Int J Behav Nutr Phys Act 11, $1-12$.

38. Metzgar C, Preston A, Miller D et al. (2015) Facilitators and barriers to weight loss and weight loss maintenance: a qualitative exploration. J Hum Nutr Diet 28, 593-603.

39. Forman EM, Goldstein SP, Crochiere RJ et al. (2019) Randomized controlled trial of ontrack, a just-in-time adaptive intervention designed to enhance weight loss. Transl Behav Med 9, 989-1001.

40. Patel ML, Hopkins CM, Brooks TL et al. (2019) Comparing self-monitoring strategies for weight loss in a smartphone app: randomized controlled trial. JMIR mHealth uHealth $\mathbf{7}$, e12209.

41. Landínez Martíneza DA, Robledo Giraldoa S \& Montoya Londoño DM (2019) Executive function performance in patients with obesity: a systematic review. Psychol Av Discip 13, 121-134.

42. Goldstein SP, Brick LA, Thomas JG et al. (2021) Examination of the relationship between lapses and weight loss in a smartphone-based just-in time adaptive intervention. Transl Behav Med 11, 993-1005.

43. Fuentes Artiles R, Staub K, Aldakak L et al. (2019) Mindful eating and common diet programs lower body weight similarly: systematic review and meta-analysis. Obes Rev 20, 1619-1627.

44. Schulze MB, Martínez-González MA, Fung TT et al. (2018) Food based dietary patterns and chronic disease prevention. BMJ 361, k2396.

45. Gardner B, Richards R, Lally P et al. (2021) Breaking habits or breaking habitual behaviours? Old habits as a neglected factor in weight loss maintenance. Appetite 162, 105183.

46. DeRigne L \& Ferrante S (2012) The sandwich generation: a review of the literature. Fla Public Health Rev 9, 12.

47. Chew HSJ, Cheng HY \& Chair SY (2019) The suitability of motivational interviewing versus cognitive behavioural interventions on improving self-care in patients with heart failure: a literature review and discussion paper. Appl Nurs Res 45, 17-22. 